\title{
Transcriptional Profiling of Sonic Hedgehog in a Prospective Cohort of Breast Cancer in a Pakistani Population
}

\author{
SYEDA KIRAN RIAZ ${ }^{1}$, LIN YE ${ }^{2}$, NAMOOD E. SAHAR ${ }^{1}$, DURKHSHAN AMAN ${ }^{1}$, JAVERIA QADIR $^{1}$, \\ JAHANGIR SARWAR KHAN $^{3}$, MUHAMMAD SAEED ${ }^{1}$, WEN G. JIANG ${ }^{3}$ and MUHAMMAD FARAZ ARSHAD MALIK ${ }^{1}$ \\ ${ }^{1}$ Department of Biosciences, COMSATS Institute of Information Technology, Islamabad, Pakistan; \\ ${ }^{2}$ Department of Surgery, Holy Family Hospital, Rawalpindi Medical College, Rawalpindi, Pakistan; \\ ${ }^{3}$ Cardiff China Medical Research Collaborative, School of Medicine, Cardiff University, Cardiff, U.K.
}

\begin{abstract}
Background/Aim: Constitutive activation of Sonic hedgehog ( $\mathrm{SHH})$ has been observed in different types of cancers. In the present study, expressional profiling of $\mathrm{SHH}$ in a breast cancer cohort $(n=150)$ of a Pakistani population and its association with different molecular subtypes have been explored. Materials and Methods: qRT-PCR and IHC were performed for expression analysis of $S H H$ and its association with ER, PR, HER2 and Ki-67 were also statistically analyzed. Results: A significant over-expression of SHH was observed in tumor tissues in comparison to their respective controls $(p<0.0001)$. A strong positive correlation was seen between SHH and proliferation marker $(r=0.635$, $p=0)$. SHH expression was significantly high among patients with advanced tumor grade, stage, nodal involvement and metastasis. Furthermore, both luminal-B and triple-negative subtypes of cohort showed increased expression of SHH. Conclusion: Based on these findings, SHH may be used as a potential biomarker for breast carcinogenesis.
\end{abstract}

Despite recent advancements in diagnosis and treatment of breast cancer, this disease is still included among the top three most common cancers affecting women (1). Hence, efforts to explore mechanistic details of carcinogenesis would facilitate in identification of potential biomarkers and hot spots for molecular therapeutics.

The Hedgehog pathway plays a crucial role in embryonic development and organogenesis. It is also considered as a key player in the development and maturation of mammary glands $(2,3)$. Sonic hedgehog $(\mathrm{SHH})$ is the most extensively

Correspondence to: Dr. Muhammad Faraz Arshad Malik, Department of Biosciences, COMSATS Institute of Information Technology, Park Road, Islamabad, Pakistan. Tel: +92 3009656522, Fax: +92 519247006, e-mail: famalik@comsats.edu.pk

Key Words: Breast cancer, Sonic hedgehog, qRT-PCR, clinicopathological features, biomarker development. studied member engaged in developmental pathway activation. Altered SHH expression profiling has already been associated with poor prognosis in various types of cancers including breast (4). Correlation of SHH overexpression with clinico-pathological parameters is a step forward towards prognostic marker development. Factors encompassing demographic data such as age at menarche, marital status and age at menopause have an impact on identification of new biomarkers. Clinico-pathological findings including grade, stage, tumor size and nodal status also influence prognostic marker development $(5,6)$. The aim of the present study was to evaluate expressional alteration of SHH in a breast cancer cohort in Pakistan using qRT-PCR and IHC. Furthermore, correlation of SHH with proliferation index (Ki-67), clinico-pathological parameters and molecular subtypes was evaluated.

\section{Materials and Methods}

Tissue collection and data retrieval. The following study was formulated and conducted after formal approval from both institutional and concerned hospital's bioethical committees respectively. Freshly excised tumors and their adjacent normal tissues ( $2 \mathrm{~cm}$ away from the macroscopic margins) were obtained from breast cancer affected patients with informed voluntary consent. These bio-specimens were immediately immersed in RNALater ${ }^{\circledR}$ solution and transported to the laboratory for further usage. Data related to age at disease onset, menarche, menopausal and marital status was also retrieved by communication. Pathological findings related to tumor grade, stage, size and nodal involvement were extracted from the concerned pathologist report and follow-up.

Extraction of RNA and cDNA synthesis. Total RNA was extracted using TRIzol ${ }^{\circledR}$ (Invitrogen, California, USA) from both tumor and control specimens. cDNA was generated using RevertAid First Strand cDNA Synthesis Kit (Thermo Scientific, California, USA) as per manufacturer's instructions. Conventional PCR was performed with $\beta$-actin primers to confirm the cDNA synthesis. Amplified products were electrophoresed on $2 \%$ agarose gel and stained with ethidium bromide for further use. 
Table I. Demographic and clinical details of the cohort along with expression of SHH and Ki-67.

\begin{tabular}{|c|c|c|c|c|c|c|c|c|c|c|c|c|c|c|c|c|c|}
\hline \multirow[t]{2}{*}{ Variable } & \multicolumn{2}{|c|}{ Age } & \multicolumn{2}{|c|}{$\begin{array}{l}\text { Menopausal } \\
\text { status }\end{array}$} & \multicolumn{3}{|c|}{ Grade } & \multicolumn{3}{|c|}{ Stage } & \multicolumn{4}{|c|}{$\begin{array}{l}\text { Tumor } \\
\text { size }\end{array}$} & \multicolumn{3}{|c|}{$\begin{array}{l}\text { Nodal } \\
\text { status }\end{array}$} \\
\hline & $\leq 45$ & $\geq 45$ & Pre- & Post & GI & GII & GIII & SI & SII & SIII & SIV & $<2 \mathrm{~cm}$ & $2-5 \mathrm{~cm}$ & $>5 \mathrm{~cm}$ & No & N1 & $\mathrm{N} 2$ \\
\hline Total (\%) & $76(51)$ & 74 (49) & $80(53)$ & $70(47)$ & $14(9)$ & $90(60)$ & $46(31)$ & $34(23)$ & $68(45)$ & $43(29)$ & $5(3)$ & $57(38)$ & $54(36)$ & $39(26)$ & $48(32)$ & $78(52)$ & $24(16)$ \\
\hline $\mathrm{SHH}^{+}$ & 63 & 44 & 65 & 40 & 7 & 73 & 38 & 21 & 47 & 37 & 4 & 35 & 36 & 35 & 22 & 68 & 20 \\
\hline $\mathrm{Ki}-67^{+}$ & 47 & 53 & 49 & 50 & 10 & 59 & 31 & 22 & 46 & 28 & 4 & 38 & 34 & 26 & 35 & 50 & 16 \\
\hline
\end{tabular}

Primer designing and $q R T-P C R$. Primers for sonic hedgehog mRNA (NM_000193.3) were designed using Integrated DNA Technology (IDT) software. Primer pair set designed for SHH amplicon (136bps) contains forward 5'-CTTCCTCACTTTCCTGGACCG-3' and reverse 5'-GGTGGCCGAGTCGTTGT-3' sequences for further processing. Target specificity of these products was confirmed with NCBI Primer Blast to avoid non-specific binding. Quantitative real time PCR (qPCR) was performed using VeriQuest SYBR Green qPCR Master Mix (Thermo Scientific, California, USA). Expression of target gene was normalized using $\beta$-actin as an internal control. The reaction condition included an initial denaturation at $95^{\circ} \mathrm{C}$ for $15 \mathrm{~min}$, followed by 40 cycles of denaturation at $95^{\circ} \mathrm{C}$ for $15 \mathrm{sec}$ and annealing at $53^{\circ} \mathrm{C}$ for $1 \mathrm{~min}$ in each cycle. Relative mRNA expression and fold change was evaluated using the $2^{-\Delta \Delta \mathrm{Ct}}$ method. Semi-quantitative reverse transcriptase PCR was also performed with same conditions as qPCR to evaluate the band intensities on $2 \%$ agarose gel.

Immunohistochemistry. Immunostaining was performed as previously described (7). Immunohistochemical staining was done using both tumor and normal frozen sections of $4 \mu \mathrm{m}$ thickness of given cohort. After fixation and blocking, these sections were treated with primary antibodies for SHH (rabbit anti-SHH polyclonal antibody, H-160; sc-9024, dilution 1:200; Santa Cruz Biotechnology, Santa Cruz, CA, USA) while an exclusion of primary antibody was employed as a negative control. The ImmunoReactive-Scores (IRS) were evaluated as the product of the \% of cells positively stained for each molecule categorized from 1 to 3 $(1=<25 \%, 2=25-50 \%, 3>50 \%)$ and intensity of staining which was scored from weak $=1$ to strong $=3$.

Statistical analysis. Data was represented as mean \pm S.D. and Wilcoxon Signed Rank test was performed to evaluate difference between tumor and control. Mann Whitney $U$-test and Kruskal Wallis Anova were applied for association of expression with clinico-pathological parameters. Furthermore, correlation between molecules was observed using Spearmen test. Graphpad Prism 5 was used to assess significance and difference was considered significant at $p<0.05$.

\section{Results}

Demographic and clinical characterization. Demographic and clinical characteristics of the cohort exhibited that mean age of breast cancer affected patients included in the study was 45 years ranging from 23 to 75 years. Out of 150 patients, $52 \%$ were $\leq 45$ years of age at the time of diagnosis. Around $30 \%$ of cohort represented poorly differentiated tumors and advance stages of breast carcinogenesis. Distribution of these details along with clinical findings and assessment of $\mathrm{SHH}$ positive samples are presented in Table I.

Relative expression of SHH among tumors compared to controls. Significant $(p<0.0001)$ up-regulation of SHH (4fold) was observed in breast tumor tissues as compared with the controls (Figures 1 and 2, Table II). Ninenty-five percent of the tumors showed positive $\mathrm{SHH}$ expression being indicative of plausible role towards cancer initiation and progression. Furthermore, strong positive association of SHH was observed with the proliferation marker Ki-67 using Spearman's rank correlation ( $\mathrm{r}=0.65, p<0.05)$. Concordance was observed between the expression of both genes and hence the role of $\mathrm{SHH}$ in tumor proliferation was established.

Correlation of hedgehog pathway with molecular subtypes of breast cancer. SHH was strongly related to the hormonal receptors estrogen receptor $(\mathrm{ER})(\mathrm{r}=0.77, p<0.05)$ and progesterone receptor $(\mathrm{PR})(\mathrm{r}=0.65, p<0.05)$ and $60 \%$ of patients were positive for both SHH and ER while it did not show any correlation with HER2. Following St. Gallen International Expert Consensus System, cohort was categorized as luminal-B (53\%), triple negative (18\%), luminal-A (15\%) and HER2 (14\%). Increased expression of $\mathrm{SHH}$ was found to be significantly associated with luminal $\mathrm{B}$ and triple negative subtypes of the cohort implicating its prognostic value in these patients (Figure 3).

Correlation of $\mathrm{SHH}$ with tumor grade and clinical stages. SHH was significantly ( $p=0.021)$ up-regulated in advanced tumors with high grade as compared to other histological grades being 3-fold higher. Distribution of $\mathrm{SHH}$ expression profiling regarding tumor differentiation is also presented in the Tables I and II. Earlier data regarding tumor size, nodal involvement and metastasis were retrieved for the given cohort. Significant up-regulation of $\mathrm{SHH}$ was observed in advanced stages (III/IV) $(p<0.0001)$. This association also related the expression of $\mathrm{SHH}$ with cancer progression due to a greater tumor size, nodal involvement and metastasis 

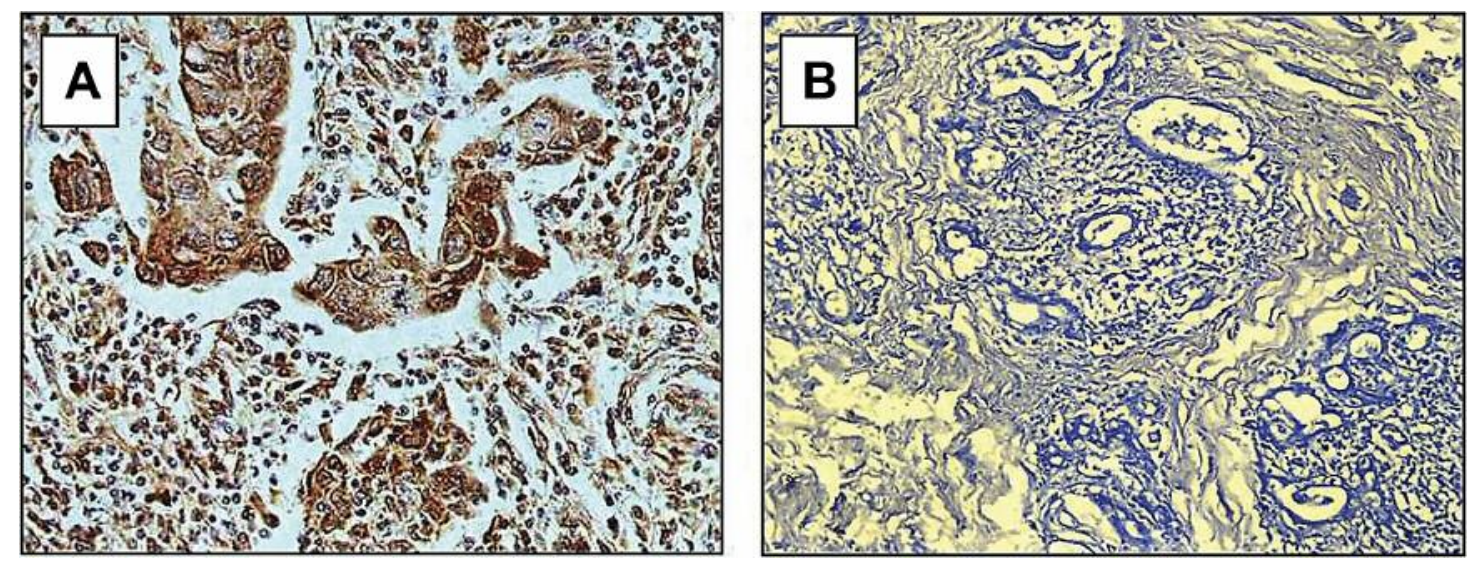

Figure 1. Immunostaining of SHH in representative breast cancer samples of cohort showing over-expression of SHH in a cytoplasmic patterncompared to adjacent normal mammary gland tissue. A) Tumor tissue and B) Normal tissue.

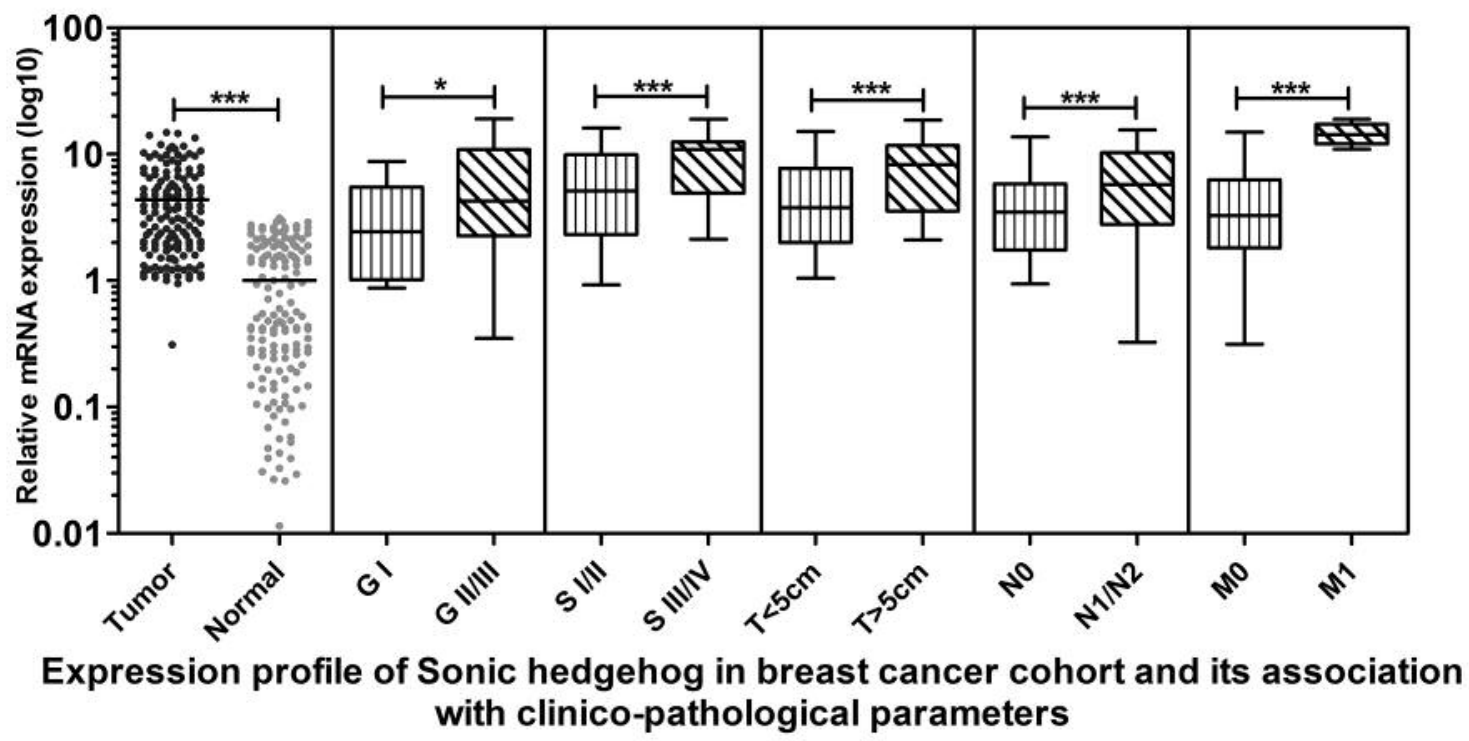

Figure 2. Distribution of SHH expression evaluated using $q P C R$ in tumor and normal tissue samples as well as its association with different clinical parameters. Plots are generated using log 10 converted median values of relative mRNA expression of SHH representing range from minimum to maximum points. $* p<0.05, * * * p<0.0001$.

which are also shown in Table II. Tumor sizes greater than $5 \mathrm{~cm}$ revealed highest number $(89.7 \%)$ with increased $\mathrm{SHH}$ expression (Table I). Involvement of lymph node metastasis was also found to be associated with $\mathrm{SHH}$ over expression having $86 \%$ patients positive for retaining nodal metastasis $(p=0.0005)$. Similarly, expression of SHH was approximately 10 -fold higher in primary tumors from patients having distant metastasis and all patients were strongly positive for SHH (Figure 2).
Correlation of SHH with age and menopausal status. Patients $\leq 45$ years of age showed significantly elevated expression of SHH $(p=0.03)$ in tumor tissues. Eighty-three percent of patients having early disease onset were positive for SHH (Table II). A similar significant correlation of SHH ( $p=0.006)$ with menopausal status of cancer affected women was observed. Expression of SHH was almost 2-fold higher and present in $81 \%$ of patients in a pre-menopausal state (Tables I and II) (Figure 3). 


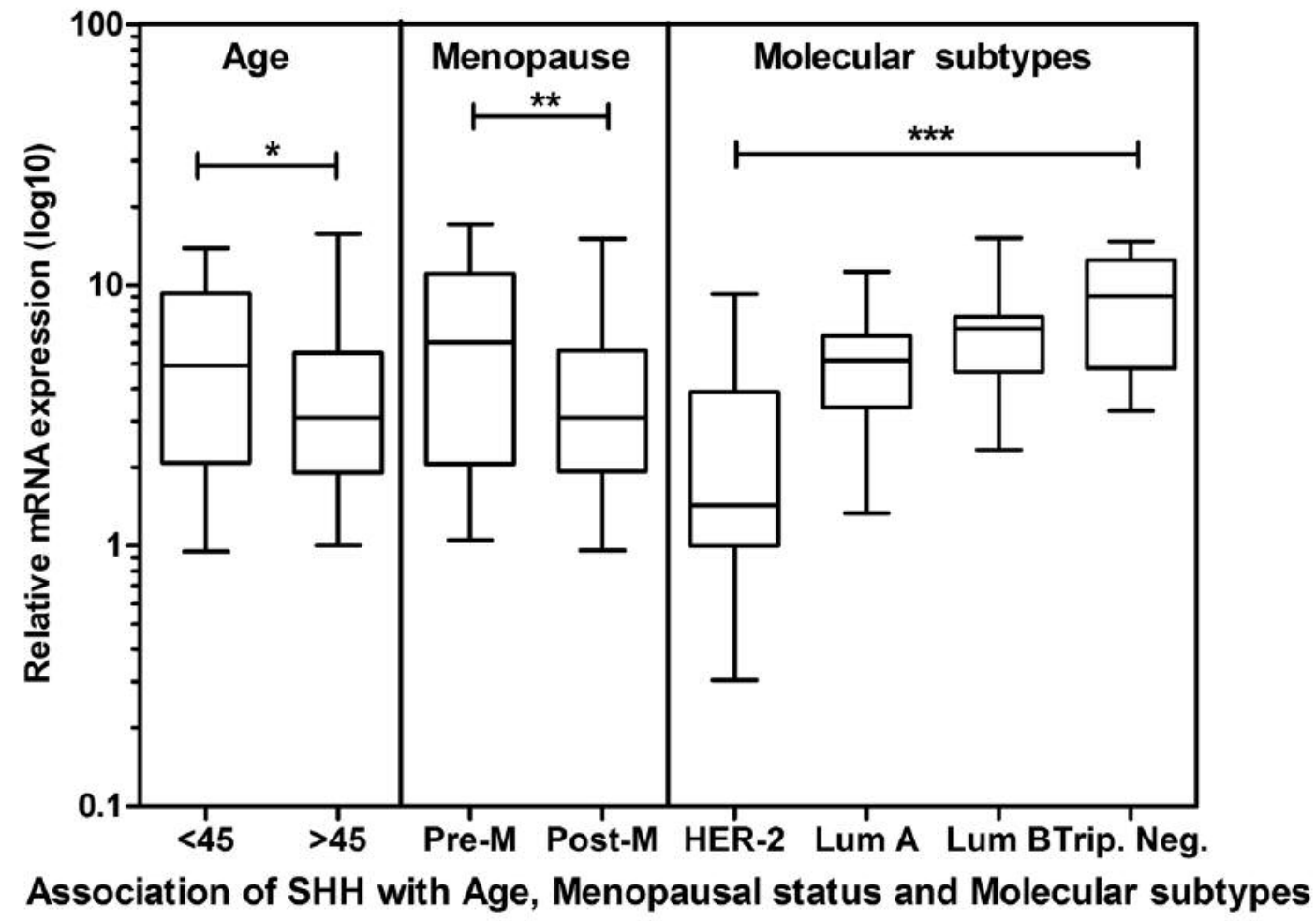

Figure 3. Box and whisker plot representing relative mRNA expression of SHH with respect to age, menopausal status and molecular subtypes. ( $q P C R$ data are shown in median values, $* p<0.05, * * p<0.001$, ***p<0.0001).

\section{Discussion}

Breast cancer is among the most common types of malignancies worldwide accounting for $23 \%$ of total cancer cases. Breast cancer incidence among Pakistani females with age-adjusted incidence rate of 69.8 per 100,000 per year is alarmingly high (1).

The Hedgehog $(\mathrm{HH})$ pathway is a key regulator of vertebrate embryonic development. It has a crucial role in cell differentiation, cell proliferation, tissue polarity and maintenance of stem cells (8). Both genetic and expressional anomalies of hedgehog members significantly influence breast carcinogenesis. Earlier, role of SHH in reprogramming cancer stem cells to aggravate different steps of breast carcinogenesis towards metastasis has also been reported (9, 10). The aim of the present study was to explore the expression profile of $\mathrm{SHH}$ in a breast cancer cohort from Pakistan. In the present study, the expression profile of sonic hedgehog was analyzed in 150 primary breast tumors along with their controls using the qRT-PCR technique. The expression profile of $\mathrm{Ki}-67$ was also evaluated in the cohort to assess the association of $\mathrm{SHH}$ with tumorigenic potential of the mammary tissues. Clinico-pathological parameters comprised of tumor size, grade, stage, nodal and distant
Table II. Correlation of SHH with clinico-pathological parameters of the cohort.

\begin{tabular}{lrrc}
\hline Variables & Total & \multicolumn{2}{c}{ SHH } \\
\cline { 3 - 4 } & & Mean \pm S.D. & $p$-Value \\
\hline Tumor & 150 & $4.4 \pm 3.3$ & $<0.0001^{*}$ \\
Control & 150 & $1.0 \pm 0.9$ & \\
Grade I & 14 & $3.4 \pm 2.7$ & $0.02^{\#}$ \\
Grade II/III & 136 & $6.5 \pm 5.3$ & \\
Stage I/II & 102 & $6.1 \pm 4.1$ & $<0.0001^{\#}$ \\
Stage III/IV & 48 & $9.3 \pm 4.3$ & \\
Tumor size <5 cm & 111 & $5.1 \pm 3.6$ & $<0.0001^{\#}$ \\
Tumor size $>5$ cm & 39 & $8.1 \pm 4.4$ & \\
No nodal involvement & 48 & $4.3 \pm 3.2$ & $0.0005^{\#}$ \\
Nodal metastasis & 102 & $6.6 \pm 4.1$ & \\
No metastasis & 145 & $4.4 \pm 3.4$ & $0.0003^{\#}$ \\
Distant metastasis & 5 & $14.6 \pm 2.9$ & \\
Age at disease onset $<45$ & 76 & $5.6 \pm 3.9$ & $0.03^{\#}$ \\
Age at disease onset $>45$ & 74 & $4.3 \pm 3.3$ & \\
Pre-menopausal status & 80 & $6.8 \pm 4.8$ & $0.0065^{\#}$ \\
Post-menopausal status & 70 & $4.5 \pm 3.5$ & \\
Laterality (left) & 75 & $6.1 \pm 4.1$ & $0.01^{\#}$ \\
Laterality (right) & 75 & $4.2 \pm 2.8$ & \\
\hline
\end{tabular}

*Wilcoxon signed rank test; ${ }^{\#}$ Mann Whitney $U$-test. 
metastasis. Age at the time of diagnosis and menopausal status was also included. Molecular subtyping of the cohort was also performed to evaluate the impact of SHH expression on patient prognostication. The majority of the samples belonged to stage II and grade II (moderately differentiated tumors) having greater tumor size and nodal metastasis.

From the given cohort, almost $95 \%$ of tumors showed significant over-expression of $\mathrm{SHH}$. These findings are consistent with earlier findings in western and Japanese populations (11, 12). SHH over-expression was analysed by using qRT-PCR in an American cohort (11) and immunohistochemistry (IHC) technique in a Japanese cohort (12). Furthermore, similar expressional variation of $\mathrm{SHH}$ was observed in a Taiwanese breast cancer cohort (4) and also in other types of cancer, such as colorectal cancers using qRT-PCR (13).

A significant correlation of SHH over expression with age was observed in the cohort. Patients having early onset possibly more frequently expressed SHH than older age participants. A contrasting trend regarding $\mathrm{SHH}$ decreased expression among elderly breast cancer affected patients had been observed (14). However, no significant association of SHH over expression and age with breast cancer incidence has also been reported (4, 15). These variations may be attributed to genetic diversity present among different tumors, population genetic makeup and cohort size. Furthermore, SHH levels among pre-menopausal women were significantly higher than the post-menopausal group. A plausible reason regarding over expression of $\mathrm{SHH}$ can be attributed towards regulation of estrogen receptor. It has been established that induction of sonic hedgehog is regulated by ER in breast cancer development (16). Association of SHH with molecular subtypes still remains unclear as very few studies have found significant relationship. Strong positive correlation was observed between SHH and ER/PR in this cohort implicating increased expression of $\mathrm{SHH}$ in patients having luminal subtypes. Also, association of $\mathrm{SHH}$ with high Ki-67 index implicates for elevated expression of SHH in luminal-B subtype in this cohort. No association was observed between HER-2 positive patients and expression of $\mathrm{SHH}$ which is contradictory to an earlier study (15). Furthermore, several studies have emphasized on the role of SHH in basal subtypes having poor prognosis and limited therapeutic options $(14,17$, 18). Increased expression of $\mathrm{SHH}$ is also correlated with the triple-negative subtype in our cohort as well implicating promising avenue for the Pakistani population.

Several groups have evaluated the clinico-pathological significance of hedgehog signalling in human breast neoplasms. Mean copy number of SHH was significantly correlated with tumor differentiation. Poorly differentiated tumors more frequently expressed this molecule than well and moderately differentiated ones in breast carcinoma. Over expression of $\mathrm{SHH}$ in poorly differentiated tumors was also observed in earlier studies in breast $(18,19)$ and gastric cancer (20). SHH expression strongly correlated with myogenic histopathological differentiation in human intestinal stromal tumors (21). However, no significant association between $\mathrm{SHH}$ expression and grades in breast cancer has been reported (22). Based on SHH having a potential role in cell differentiation, validation using in vivo modelling is proposed to authenticate this correlation.

Expressional regulation of $\mathrm{SHH}$ was 3-fold higher in advanced stages in breast cancer patients in Pakistan. Eighty six percent positive samples in stage III/IV clearly indicated significant correlation of $\mathrm{SHH}$ with advanced staging. SHH levels were 3-fold higher among patients with tumor size $>5 \mathrm{~cm}$. Furthermore, elevated SHH levels were observed in a cohort with lymph node involvement and distant metastasis. These findings were also in line with previously published reports $(15,22)$. Earlier, high expression of SHH was directly correlated with tumor stages but lacked any significant association with nodal involvement and metastasis $(4,18)$.

Apart from data generated concerning Pakistani women affected by breast cancer, current findings are also in line with earlier studies $(11,19)$. The results are in coherence with reports using qRT-PCR techniques on fresh tumor tissue samples of breast (4) and colon (13) cancers. The results at both mRNA and protein levels were consistent in this study. Assessment of SHH using qRT-PCR is a more rapid and sensitive technique. Evaluation using IHC may only provide ambiguous information due to different antibodies used for SHH detection. It is suggested to validate the results at both mRNA and protein levels to provide reliable therapeutic options. These results support the already reported clinico-pathological relevance of $\mathrm{SHH}$ for biomarker development. It is proposed to use fresh biopsy samples for assessment of SHH expression along with GLI-1 for better prognostication of breast cancer patients. This strategy may prove to be vital for therapeutic decisions in clinical application of breast cancer treatment.

\section{Acknowledgements}

This study was funded by National research program for universities (NRPU) of the Higher Education Commission of Pakistan project ID 2989.

\section{Conflicts of Interest}

The Authors of the study have no conflicts of interest.

\section{References}

1 Torre LA, Bray F, Siegel RL, Ferlay J, Lortet-Tieulent J and Jemal A: Global cancer statistics, 2012. CA Cancer J Clin 65: 87-108, 2015.

2 Hui M, Cazet A, Nair R, Watkins DN, O'Toole SA and Swarbrick A: The Hedgehog signalling pathway in breast development, carcinogenesis and cancer therapy. Breast Cancer Res 15: 203, 2013. 
3 Moraes RC, Chang H, Harrington N, Landua JD, Prigge JT, Lane TF, Wainwright BJ, Hamel PA and Lewis MT: Ptch1 is required locally for mammary gland morphogenesis and systemically for ductal elongation. Development 136: 14231432, 2009.

4 Jeng K-S, Yu M-C, Hsiau H-I, Chang F-Y, Sheen I-S and Jeng W-J: High expression of Sonic Hedgehog signaling pathway genes indicates a risk of recurrence of breast carcinoma. Onco Targets Ther 7: 79, 2013.

5 Weigelt B, Peterse JL and van't Veer LJ: Breast cancer metastasis: markers and models. Nat Rev Cancer 5: 591-602, 2005.

6 Cianfrocca M: Prognostic and predictive factors in early-stage breast cancer. Oncologist 9: 606-616, 2004.

7 Malik FA, Sanders AJ, Jones AD, Mansel RE and Jiang WG: Transcriptional and translational modulation of KAI1 expression in ductal carcinoma of the breast and the prognostic significance. Int J Mol Med 23: 273-278, 2009.

8 Gupta S, Takebe N and Lorusso P: Targeting the Hedgehog pathway in cancer. Ther Adv Med Oncol 2: 237-250, 2010.

9 Cui W, Wang L-H, Wen Y-Y, Song M, Li B-L, Chen X-L, Xu M, An S-X, Zhao J, Lu Y-Y, Mi X-Y and Wang E-H: Expression and regulation mechanisms of Sonic Hedgehog in breast cancer. Cancer Sci 101: 927-933, 2010.

10 Ruiz i Altaba A: Hedgehog signaling and the Gli code in stem cells, cancer, and metastases. Sci Signal 4: pt9, 2011.

11 Mukherjee S, Frolova N, Sadlonova A, Novak Z, Steg A, Page GP, Welch DR, Lobo-Ruppert SM, Ruppert JM, Johnson MR and Frost AR: Hedgehog signaling and response to cyclopamine differ in epithelial and stromal cells in benign breast and breast cancer. Cancer Biol Ther 5: 674-683, 2006.

12 Kubo M: Hedgehog Signaling Pathway is a New Therapeutic Target for Patients with Breast Cancer. Cancer Res 64: 60716074, 2004.

13 Monzo M, Moreno I, Artells R, Ibeas R, Navarro A, Moreno J, Hernandez R, Granell M and Pie J: Sonic hedgehog mRNA expression by real-time quantitative PCR in normal and tumor tissues from colorectal cancer patients. Cancer Lett 233: 117$123,2006$.

14 Noman AS, Uddin M, Rahman MZ, Nayeem MJ, Alam SS, Khatun Z, Wahiduzzaman M, Sultana A, Rahman ML, Ali MY, Barua D, Ahmed I, Islam MS, Aboussekhra A, Yeger H, Farhat WA and Islam SS: Overexpression of sonic hedgehog in the triple negative breast cancer: clinicopathological characteristics of high burden breast cancer patients from Bangladesh. Sci Rep 6: 18830, 2016.
15 Im S, Choi HJ, Yoo C, Jung J-H, Jeon Y-W, Suh YJ and Kang CS: Hedgehog related protein expression in breast cancer: gli-2 is associated with poor overall survival. Korean J Pathol 47: 116-123, 2013.

16 Koga K, Nakamura M, Nakashima H, Akiyoshi T, Kubo M, Sato N, Kuroki S, Nomura M, Tanaka M and Katano M: Novel link between estrogen receptor alpha and hedgehog pathway in breast cancer. Anticancer Res 28: 731-740, 2008.

17 Flemban A and Qualtrough D: The Potential Role of Hedgehog Signaling in the Luminal/Basal Phenotype of Breast Epithelia and in Breast Cancer Invasion and Metastasis. Cancers (Basel) 7: 1863-1884, 2015.

18 Tao Y, Mao J, Zhang Q and Li L: Overexpression of Hedgehog signaling molecules and its involvement in triple-negative breast cancer. Oncol Lett 2: 995-1001, 2011.

19 O'Toole SA, Machalek DA, Shearer RF, Millar EK, Nair R, Schofield P, McLeod D, Cooper CL, McNeil CM, McFarland A, Nguyen A, Ormandy CJ, Qiu MR, Rabinovich B, Martelotto LG, Vu D, Hannigan GE, Musgrove EA, Christ D, Sutherland RL, Watkins DN and Swarbrick A: Hedgehog overexpression is associated with stromal interactions and predicts for poor outcome in breast cancer. Cancer Res 71: 4002-4014, 2011.

20 Niu Y, Li F, Tang B, Shi Y, Hao Y and Yu P: Clinicopathological correlation and prognostic significance of sonic hedgehog protein overexpression in human gastric cancer. Int J Clin Exp Pathol 7: 5144-5153, 2014.

21 Yoshizaki A, Nakayama T, Naito S, Wen CY and Sekine I: Expressions of sonic hedgehog, patched, smoothened and Gli-1 in human intestinal stromal tumors and their correlation with prognosis. World J Gastroenterol 12: 5687-5691, 2006.

22 Souzaki M, Kubo M, Kai M, Kameda C, Tanaka H, Taguchi T, Tanaka M, Onishi $\mathrm{H}$ and Katano M: Hedgehog signaling pathway mediates the progression of non-invasive breast cancer to invasive breast cancer. Cancer Sci 102: 373-381, 2011.
Received May 19, 2017

Revised June 12, 2017

Accepted June 13, 2017 